\title{
Dimensiones de la apófisis pterigoides en pacientes con $y$ sin disfunción Temporomandibular.
}

\author{
Dimensions of the pterygoid process in patients with and without temporomandibular dysfunction.
}

Jorge Beltrán-Silva ${ }^{1, a}$, Katty Ríos-Villasis ${ }^{2, b}$, Hugo Ronquillo-Herrera ${ }^{3, c}$, Alexis Evangelista-Alva $^{1, b}$.

\section{RESUMEN}

Introducción: La función muscular condiciona cambios dinámicos en la orientación de sus fibras y posteriormente en su longitud real; estos cambios tensionales producen una remodelación en la estructura ósea interna del hueso de inserción y luego de un período de tiempo pueden condicionar cambios significativos en la arquitectura del hueso donde actúan. Objetivo: comparar las dimensiones del ala externa de la apófisis pterigoides a través de tomografía de haz cónico en pacientes con y sin disfunción temporomandibular. Materiales y métodos: se evaluaron 60 tomografías de haz cónico de pacientes con disfunción temporomandibular y 60 tomografías de pacientes sin la condición, entre los años 2010 y 2011. Resultados: se estableció que la longitud media del ala externa de la apófisis pterigoides, en pacientes con disfunción temporomandibular fue de 17,01 $\pm 3,64 \mathrm{~mm}$ en el lado derecho y de $16,21 \pm 3,51 \mathrm{~mm}$ en el lado izquierdo; y en pacientes $\sin$ disfunción temporomandibular fue de $11,86 \pm 1,97 \mathrm{~mm}$ en el lado derecho y $11,98 \pm 1,85 \mathrm{~mm}$ en el lado izquierdo. Además se encontró relación estadísticamente significativa entre el lado de mayor longitud del ala externa de la apófisis pterigoides y dolor ipsilateral $(\mathrm{p}<0,05)$. Conclusiones: existen diferencias altamente significativas en la longitud del ala externa de la apófisis pterigoides, medida en tomografía de haz cónico, entre los pacientes con y sin disfunción temporomandibular $(\mathrm{p}<0,001)$.

\section{Palabras clave: ARTICULACIÓN TEMPOROMANDIBULAR, HUESO ESFENOIDES, MANDÍBULA.}

\section{ABSTRACT}

Introduction: Muscular function determines the dynamic changes in orientation of its fibers and later in its length, these pressure changes occur in the structure remodeling bone marrow insertion internal and after a period of time may determine significant changes in the architecture bone where they act. Objectives: To compare lateral plate's dimensions of pterygoid process (LPPP) by cone beam computer tomography (CBCT) in patients with and without temporomandibular dysfunction (TMD). Material and methods: 60 CBCT of patients with TMD and 60 CBCT patients without that condition, from 2010 to 2011. Results: Results showed patients with larger LPPP and with TMD were $17.01 \pm 3.64 \mathrm{~mm}$ on right side and 16.21

\footnotetext{
1 International Association of DentoMaxilofacial Radiology. Tygerberg, South Africa.

${ }^{2}$ Facultad de Odontología. Universidad Inca Garcilaso de la Vega. Lima, Perú.

3 Facultad de Estomatología. Universidad Peruana Cayetano Heredia. Lima, Perú.

a Magíster en Estomatología. Especialista en Radiología Oral y Maxilofacial.

b Magíster en Estomatología.

c Magíster en Estomatología. Especialista en Rehabilitación Oral.
} 
$\pm 3.51 \mathrm{~mm}$ on left side; and in patients without TMD were $11.86 \pm 1.97 \mathrm{~mm}$ on right side and $11.98 \pm 1.85 \mathrm{~mm}$ on left side. We found statistically significant relation between the side of major length of LPPP and pain in the same side $(p<0.05)$. Conclusions: There was highly significant differences in LPPP length, measured in CBCT, between patients with and without TMD $(p<0.001)$.

Key words: TEMPOROMANDIBULAR JOINT, SPHENOID BONE, MANDIBLE.

\section{INTRODUCCIÓN}

Los trastornos que afectan a la articulación temporomandibular (ATM) son de índole multifactorial porque involucran aspectos de tipo afectivo-emocional, morfo-fisiología de los músculos del macizo facial, disposición anatómico funcional de la columna cervical, componente dentoalveolar con énfasis en la armonía oclusal y la propia morfología y función de la ATM. Existen diversas literaturas que demuestran alteraciones de crecimiento a nivel mandibular o maxilar que se pueden compatibilizar con hiperplasia o hipoplasia. Sin embargo, el hueso esfenoides de por sí no reporta un síndrome o alteración directa que pueda alterar sólo sus apófisis pterigoides $(1,2)$. Cuando los pacientes sufren hiperplasia hemifacial se produce un aumento en las dimensiones de los huesos faciales de la mitad de la cara, sin embargo la zona central de la base craneal no se altera en sus dimensiones transversales $(1,2)$.

La función muscular condiciona cambios dinámicos en la orientación de sus fibras y posteriormente en su longitud real. Estos cambios tensionales producen una remodelación en la estructura ósea interna del hueso de inserción y luego de un período de tiempo aún no establecido, pueden condicionar cambios significativos en la arquitectura del hueso donde actúan $(3,4)$. El aspecto morfológico de varias estructuras del macizo facial está involucrado en el desarrollo de problemas de la ATM. Es así que, la observación continua en nuestra práctica privada, a través de tomografía de haz cónico, de asimetrías entre los componentes óseos del ala externa de la apófisis pterigoides en pacientes con trastornos de ATM nos dejó una gran pregunta. ¿Existirá relación entre esta asimetría morfológica de la apófisis pterigoides con el desarrollo de problemas en las ATM? Por ende, el propósito del estudio fue comparar las dimensiones del ala externa de la apófisis pterigoides a través de tomografía Cone Beam en pacientes con y sin disfunción temporomandibular.

\section{MATERIAL Y MÉTODOS}

Se realizó un estudio observacional, prospectivo, transversal y comparativo. Previamente se solicitó la autorización al Comité Institucional de Ética para Humanos de la Universidad Peruana Cayetano Heredia, siendo el estudio aprobado con código SIDISI 57819. Para el cálculo del tamaño muestral se aplicó la fórmula para comparación de dos medias; el tipo de muestreo fue no probabilístico; la muestra estuvo conformada por 60 pacientes con Disfunción Temporomandibular (DTM) y 60 pacientes sin la condición, de ambos sexos y mayores de edad (mayor de 18 años) que acudieron al centro privado de radiología oral y maxilofacial IMÁGENES, para realizarse la evaluación por tomografía computarizada Cone Beam, y que cumplían con los criterios de inclusión y exclusión del estudio; la distribución de la muestra se hizo según grupo etáreo: Menores de 20 años (18 a 20 años), Adultos jóvenes (21 a 30 años), Adultos (31 a 40 años); Adultos premayores (41 a 50 años ) y Adulto mayor ( 51 a más años).

A cada individuo que cumplía con los criterios establecidos para el estudio, se le brindó información del mismo y voluntariamente daban su aprobación a través del consentimiento informado. Luego, se le tomó una tomografía computarizada de haz cónico (Cone Beam) en un equipo Icat Next Generation (Kavo, Hartschein, USA). Para esto, cada sujeto se sentó en el equipo con la columna vertebral erguida y la cabeza apoyada en un soporte posterior. Todo paciente llevó un delantal plomado que lo cubría en la región cervical y torácica. Una vez ubicado se colocó un soporte de mentón que lo fija y mantiene estable. Todas las adquisiciones se realizaron en mordida habitual, con el plano oclusal paralelo al piso y la línea media centrada con respecto a su eje principal. El equipo realizó un giro de $360^{\circ}$ alrededor del paciente en un tiempo de 26,9 segundos y con una resolución de $0,25 \mathrm{~mm}$ de voxel size. La dosis de radiación promedio fue de $0.087 \mathrm{mSv}$ lo que equivale a once días 
de radiación natural o el aproximado de exposición que se recibe por un vuelo entre Los Ángeles y Londres(5). El paciente fue instruído para que durante el examen permanezca quieto, sin pasar saliva y con una respiración suave. Las tomografías computarizadas de haz cónico (Cone Beam) de los individuos se recolectaron de la base de datos del centro privado. Se procedió a parear la muestra en edad y sexo entre ambos grupos de estudio, pacientes con disfunción temporomandibular y pacientes sanos.

Una vez obtenida la tomografía en el software IcatVision (Kavo, Hartschein, USA) se exploró la pantalla de reconstrucción multiplanar MPR. Se ubicó el plano sagital medio y el eje axial sobre el plano palatino para poder obtener una visualización axial apropiada de las alas externas de la apófisis pterigoides bilateral.

Las medidas de longitud se realizaron sobre el plano axial, tomando como referencia el plano del piso de fosas nasales, desde el extremo más distal del ala externa de la apófisis pterigoides hasta el punto de unión entre el ala externa e interna. Las medidas se realizaron en milímetros con dos decimales, en ambos lados y se ingresaron a la ficha de recolección de datos.

Para el análisis estadístico de los resultados se utilizó el programa SPSS 18,0 ${ }^{\circledR}$ para Windows. En este trabajo de investigación se fijó un grado de significancia de 0,05 que corresponde a un intervalo de confianza del $95 \%$, y un poder de la prueba del $80 \%$. Las variables cualitativas se describieron expresando el porcentaje de cada categoría. Las variables cuantitativas se describieron utilizando la media aritmética y la desviación estándar. Se efectuó la prueba de Kolmogorov - Smirnoff y se aceptó la normalidad si $p>0,05$. Cuando teníamos variables cuantitativas con distribución normal se realizó pruebas paramétricas como t-student para muestras independientes.

\section{RESULTADOS}

Se analizaron 120 tomografías computarizadas Cone Beam de 60 pacientes con disfunción temporomandibular y 60 sin disfunción. Los 60 pacientes sin disfunción temporomandibular tenían la misma edad y sexo que el otro grupo, lo que permitió tener una muestra homogénea. Se obtuvo una media del ala ex- terna de la apófisis pterigoides derecha en pacientes con disfunción fue de 17,01 $\mathrm{mm} \pm 3,64$. La media del lado izquierdo en este grupo fue de 16,21 $\mathrm{mm} \pm 3,51$. La media del ala externa de la apófisis pterigoides derecha en pacientes sin disfunción fue de $11,86 \mathrm{~mm} \pm$ 1,97. La media del lado izquierdo en este grupo fue de $11,98 \mathrm{~mm} \pm 1,85$. Al comparar la longitud del ala externa de la apófisis pterigoides entre los pacientes con y sin disfunción temporomandibular, se encontró que habían diferencias estadísticamente significativas tanto en el lado derecho $(\mathrm{p}<0,001)$ como en el lado izquierdo $(\mathrm{p}<0,001)$, como se puede observar en la tabla 1 y en el gráfico 1 . La media de la edad en los pacientes con disfunción temporomandibular fue de $40,10 \pm 15,43$. La media de la edad en los pacientes sin disfunción temporomandibular fue de 40,02 \pm 15,38 .

Tabla 1. Comparación de la longitud del ala externa de la apófisis pterigoides entre los pacientes con y sin Disfunción Temporomandibular.

\begin{tabular}{lcccc}
\hline \multicolumn{1}{c}{ Condición } & n & Media $^{+}$ & DS & $\boldsymbol{p}^{*}$ \\
\hline LADO DERECHO & & & & \\
PACIENTES CON DCM & 60 & 17,01 & 3,64 & \\
& & & & $<0,001$ \\
PACIENTES SIN DCM & 60 & 11,86 & 1,96 & \\
LADO IZQUIERDO & & & & \\
PACIENTES CON DCM & 60 & 16,21 & 3,50 & \\
& & & & $<0,001$ \\
PACIENTES SIN DCM & 60 & 11,97 & 1,85 & \\
\hline
\end{tabular}

DCM=Disfunción temporomandibular; DS=Desviación Estándar.

* Prueba t-student para muestras independientes.

+ Medidas en mm

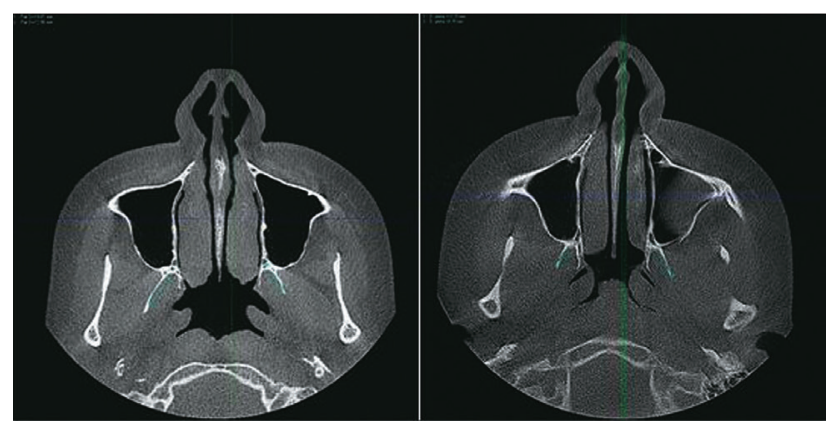

Grafico 1. Paciente control y paciente con Disfunción temporomandibular. 
En el grupo de menores de 20 años con disfunción temporomandibular se encontró una longitud media del ala externa de la apófisis pterigoides del lado derecho de $16.35 \mathrm{~mm} \pm 4.53$. En el lado izquierdo la longitud promedio fue de $15,78 \mathrm{~mm} \pm 3,07$. En el grupo de menores de 20 años sin disfunción temporomandibular se encontró una longitud media del ala externa de la apófisis pterigoides del lado derecho de $12,39 \mathrm{~mm} \pm 2,55$. En el lado izquierdo la longitud promedio fue de $12,28 \mathrm{~mm} \pm 2,29$. Al comparar la longitud del ala externa de la apófisis pterigoides entre los pacientes con y sin disfunción temporomandibular del grupo de menores de 20 años, se encontró que no habían diferencias estadísticamente significativas en el lado derecho ( $p>0,05)$, pero en el lado izquierdo si existía diferencia estadísticamente significativa $(\mathrm{p}<0,05)$.En el grupo de adultos jóvenes con disfunción temporomandibular se encontró una longitud media del ala externa de la apófisis pterigoides del lado derecho de $16,27 \mathrm{~mm} \pm 3,68$. En el lado izquierdo la longitud promedio fue de $16,44 \mathrm{~mm} \pm 2,97$. En el grupo de adultos jóvenes sin disfunción temporomandibular se encontró una longitud media del ala externa de la apófisis pterigoides del lado derecho de $12,70 \mathrm{~mm} \pm 2,13$. En el lado izquierdo la longitud promedio fue de $12,84 \mathrm{~mm} \pm 2,30$. Al comparar la longitud del ala externa de la apófisis pterigoides entre los pacientes con y sin disfunción temporomandibular del grupo adultos jóvenes, se encontró que habían diferencias estadísticamente significativas en el lado derecho $(p<0,05)$ y lado izquierdo $(p<0,05)$.En el grupo de adultos con disfunción temporomandibular se encontró una longitud media del ala externa de la apófisis pterigoides del lado derecho de $16,12 \mathrm{~mm} \pm$ 2,36. En el lado izquierdo la longitud promedio fue de $16,63 \mathrm{~mm} \pm 4,06$. En el grupo de adultos sin disfunción temporomandibular se encontró una longitud media del ala externa de la apófisis pterigoides del lado derecho de $11,72 \mathrm{~mm} \pm 2,04$. En el lado izquierdo la longitud promedio fue de $11,84 \mathrm{~mm} \pm 1,77$. Al comparar la longitud del ala externa de la apófisis pterigoides entre los pacientes con y sin disfunción temporomandibular del grupo de adultos, se encontró que habían diferencias estadísticas altamente significativas en el lado derecho $(\mathrm{p}<0,05)$ y lado izquierdo $(\mathrm{p}<0,05)$.

En el grupo de adultos premayores con disfunción temporomandibular se encontró una longitud media del ala externa de la apófisis pterigoides del lado derecho de $18.28 \mathrm{~mm} \pm 3.24$. En el lado izquierdo la longitud promedio fue de $16,30 \mathrm{~mm} \pm 3,74$. En el grupo de adultos premayores sin disfunción temporomandibular se encontró una longitud media del ala externa de la apófisis pterigoides del lado derecho de $11,72 \mathrm{~mm} \pm 1,79$. En el lado izquierdo la longitud promedio fue de $11,74 \mathrm{~mm} \pm 1,60$. Al comparar la longitud del ala externa de la apófisis pterigoides entre los pacientes con y sin disfunción temporomandibular del grupo adultos premayores, se encontró que habían diferencias estadísticas altamente significativas en el lado derecho $(\mathrm{p}<0,05)$ y lado izquierdo $(\mathrm{p}=0,05)$ como se puede observa en la tabla 2.En el grupo de adultos mayores con disfunción temporomandibular se encontró una longitud media del ala externa de la apófisis pterigoides del lado derecho de $17,71 \mathrm{~mm}$ $\pm 4,57$. En el lado izquierdo la longitud promedio fue de $15,73 \mathrm{~mm} \pm 3,67$. En el grupo de adultos mayores sin disfunción temporomandibular se encontró una longitud media del ala externa de la apófisis pterigoides del lado derecho de $11,24 \mathrm{~mm} \pm 1,57$. En el lado izquierdo la longitud promedio fue de $11,52 \mathrm{~mm} \pm$ 1,55. Al comparar la longitud del ala externa de la apófisis pterigoides entre los pacientes con y sin disfunción temporomandibular del grupo adultos mayores, se encontró que habían diferencias estadísticas altamente significativas en el lado derecho $(p<0,05)$ y lado izquierdo $(p<0,05)$ como se puede observa en la tabla 3.

Tabla 2. Comparación de la longitud del ala externa de la apófisis pterigoides entre los pacientes con y sin disfunción temporomandibular del grupo adultos premayores.

\begin{tabular}{lcccc}
\hline \multicolumn{1}{c}{ Condición } & n & Media $^{+}$ & DS & $\boldsymbol{p}^{*}$ \\
\hline LADO DERECHO & & & & \\
PACIENTES CON DCM & 13 & 18,28 & 3,24 & \\
& & & & $<0,001$ \\
PACIENTES SIN DCM & 13 & 11,72 & 1,79 &
\end{tabular}

\section{LADO IZQUIERDO}

\begin{tabular}{lcccc} 
PACIENTES CON DCM & 13 & 16,30 & 3,74 & \\
& & & & 0,001 \\
PACIENTES SIN DCM & 13 & 11,74 & 1,60 & \\
\hline
\end{tabular}

Grupo de edad = adulto pre-mayor

DCM=Disfunción temporomandibular; DS=Desviación Estándar.

* Prueba t-student para muestras independientes.

+ Medidas en mm 
Tabla 3. Comparación de la longitud del ala externa de la apófisis pterigoides entre los pacientes con y sin disfunción temporomandibular del grupo adultos mayores.

\begin{tabular}{lcccc}
\hline \multicolumn{1}{c}{ Condición } & n & Media $^{+}$ & DS & $\boldsymbol{p}^{*}$ \\
\hline LADO DERECHO & & & & \\
PACIENTES CON DCM & 14 & 17,71 & 4,57 & \\
& & & & $<0,001$ \\
PACIENTES SIN DCM & 14 & 11,24 & 1,57 & \\
LADO IZQUIERDO & & & & \\
PACIENTES CON DCM & 14 & 15,73 & 3,67 & \\
& & & & 0,001 \\
PACIENTES SIN DCM & 14 & 11,52 & 1,55 & \\
\hline
\end{tabular}

Grupo de edad = adulto mayor

DCM=Disfunción temporomandibular; DS=Desviación Estándar.

* Prueba t-student para muestras independientes.

+ Medidas en mm

En los pacientes con disfunción temporomandibular y dolor en el lado derecho se encontró una longitud media del ala externa de la apófisis pterigoides del lado derecho de $18,58 \mathrm{~mm} \pm 3,32$. En el lado izquierdo la longitud promedio fue de $14,65 \mathrm{~mm} \pm 3,28$. Se encontró una relación estadística altamente significativa $(p<0,05)$ entre la longitud del ala externa de la apófisis pterigoides del lado derecho y el dolor en el mismo lado.

En los pacientes con disfunción temporomandibular y dolor en el lado izquierdo se encontró una longitud media del ala externa de la apófisis pterigoides del lado derecho de $15,14 \mathrm{~mm} \pm 3,21$. En el lado izquierdo la longitud promedio fue de $18,35 \mathrm{~mm} \pm 3,38$. Se encontró una relación estadísticamente significativa $(\mathrm{p}<0,05)$ entre la longitud del ala externa de la apófisis pterigoides del lado izquierdo y el dolor en el mismo lado; como se puede observa en la tabla 4.

En los pacientes con disfunción temporomandibular y dolor bilateral se encontró una longitud media del ala externa de la apófisis pterigoides del lado derecho de $17,27 \mathrm{~mm} \pm 3,67$. En el lado izquierdo la longitud promedio fue de $15,53 \mathrm{~mm} \pm 2,35$. No se encontró una relación estadísticamente significativa ( $p>0,05)$ entre la longitud del ala externa de la apófisis pterigoides y el dolor bilateral; como se puede observa en la tabla 5 .
Tabla 4. Comparación entre la longitud del ala externa de la apófisis pterigoides y presencia de dolor en el lado izquierdo en pacientes con DCM.

\begin{tabular}{lllll}
\hline \multicolumn{1}{c}{ Condición } & $\mathbf{N}$ & Media $^{+}$ & DS & $\boldsymbol{p}^{*}$ \\
\hline PACIENTES CON DCM & & & & \\
Y DOLOR EN LADO & & & & \\
IZQUIERDO & & & &
\end{tabular}

\begin{tabular}{lllll} 
LADO DERECHO & 22 & 15,14 & 3,21 & \\
& & & & 0,002 \\
LADO IZQUIERDO & 22 & 18,35 & 3,38 & \\
\hline
\end{tabular}

Condición $=$ DOLOR LADO IZQUIERDO

DCM=Disfunción temporomandibular; DS=Desviación Estándar.

* Prueba t-student para muestras independientes.

+ Medidas en mm

Tabla 5. Comparación entre la longitud del ala externa de la apófisis pterigoides y presencia de dolor bilateral en pacientes con DCM.

\begin{tabular}{ccccc}
\hline Condición & N & Media $^{+}$ & DS & $p^{*}$ \\
\hline PACIENTES CON DCM & & & & \\
Y DOLOR BILATERAL & & & &
\end{tabular}

$\begin{array}{lcccc}\text { LADO DERECHO } & 14 & 17,27 & 3,67 & \\ & & & & 0,148 \\ \text { LADO IZQUIERDO } & 14 & 15,53 & 2,35 & \end{array}$

Condición $=$ DOLOR BILATERAL

DCM=Disfunción temporomandibular; DS=Desviación Estándar.

* Prueba t-student para muestras independientes.

+ Medidas en mm

\section{DISCUSIÓN}

Los sistemas de tomografía de haz cónico o Cone Beam son en la actualidad utilizados directamente por los odontólogos, lo que nos permite evaluar detalles críticos para nuestras especialidades y encontrar hallazgos que nos pueden guiar a nuevas investigaciones para responder a interrogantes diferentes a las tradicionales. Esta investigación es parte de esta nueva tendencia, ya que nació de una secuencia de observaciones directas de tomografías en las cuales evidenciamos diferencias entre las longitudes del ala externa de la apófisis pterigoides. 
La muestra contó con 120 sujetos, de los cuales 60 tenían disfunción temporomandibular y los otros 60 sin esa condición. Se buscó una muestra homogénea, para lo cual se igualó las edades y sexos de los casos con los pacientes sin disfunción. Esto se evidenció al realizar la prueba estadística de Kolmogorov-Smirnoff $(\mathrm{p}>0,05)$, la cual arrojó que nuestra muestra por cada grupo, tenía distribución normal y en la comparación por promedios de edad, fue similar en ambos grupos.

En el presente estudio, la media del ala externa de la apófisis pterigoides en pacientes con disfunción temporomandibular fue en el lado derecho $17,01 \mathrm{~mm}$ con desviación estandar de 3,64 y en el lado izquierdo $16,21 \mathrm{~mm}$ con desviación estandar de 3,51. Ueki K. evaluó 82 pacientes japoneses con prognatismo mandibular y asimetría facial, encontrando una media en la longitud del ala externa de la apófisis pterigoides para el lado derecho de $11,3 \mathrm{~mm}$ con desviación estandar de 3,2 y para el lado izquierdo una media de $12,6 \mathrm{~mm}$ con una desviación estandar de 3,9 (6). Estos datos son diferentes, pero si la comparación la realizamos con nuestros datos de pacientes sin disfunción temporomandibular vamos a encontrar una similitud importante.

La media en el presente estudio del ala externa de la apófisis pterigoides derecha en pacientes sin disfunción fue de $11,86 \mathrm{~mm}$ con desviación estandar de 1,97 y en el lado izquierdo fue 11,98mm con desviación estandar de 1,85. Estos datos son comparables con los reportados por Ueki K (6), y refuerza la diferencia que existe entre ambos grupos, ya que los pacientes que utilizó el autor no tenían disfunción temporomandibular reportada.

En esta investigación, al comparar la longitud del ala externa de la apófisis pterigoides entre los pacientes con y sin disfunción temporomandibular, se encontró que habían diferencias estadísticamente significativas tanto en el lado derecho $(p<0,001)$ como en el lado izquierdo $(p<0,001)$. Este dato encontrado en la presente investigación no ha sido reportado hasta el momento en la literatura nacional o internacional.

La hiperactividad muscular condiciona cambios dinámicos en la orientación de sus fibras y posteriormente en su longitud real. Estos cambios tensionales producen remodelación en la estructura ósea de in- serción, pudiendo condicionar cambios significativos en la arquitectura del hueso, luego de un período de tiempo aún no establecido $(3,4)$.

La hiperactividad del músculo pterigoideo lateral puede causar pequeñas injurias en la estructura articular (1). Gidarakou sugirió que la enfermedad degenerativa articular puede afectar la morfología esquelética (7). Trpkova reportó que las mujeres con trastorno degenerativo de la articulación temporomandibular pueden presentar o desarrollar discrepancias mandibulares de tipo vertical (8).

Al producirse una disfunción de la articulación temporomandibular, se establece una degeneración interna, que normalmente se inicia con un desplazamiento del disco articular. Este desplazamiento puede ser causado por un trauma o una injuria leve permanente que condiciona un espasmo de largo tiempo en el músculo pterigoideo lateral, predominantemente hacia su fascículo superior que se inserta principalmente en el ala externa de la apófisis pterigoides y el polo anteromedial del disco articular (9).

La observación empírica que dio origen a este estudio, se confirma con los resultados obtenidos y posiblemente se explicaría porque los pacientes con disfunción temporomandibular tienen un espasmo crónico del músculo pterigoideo lateral; esta excesiva tensión muscular produce una remodelación progresiva del ala externa de la apófisis pterigoides, que condicionaría su alargamiento en un lapso de tiempo no determinado.

Los resultados que encontramos al relacionar el grupo etario, con respecto a la longitud del ala externa de la apófisis pterigoides en pacientes con y sin disfunción de ATM, nos mostraron diferencias estadísticamente significativos en los grupos de jóvenes, adultos, adultos premayores y adultos mayores $(p<0,05)$ en ambos lados. Sólo el grupo de menores de 20 años en el lado derecho demostró que no habían diferencias estadísticamente significativas $(p=0,067)$, lo cual se podría atribuir a que aún no han concluído su crecimiento.

Al realizar las comparaciones por sexo entre los grupos con la condición y sin ella, no se encontró diferencias estadísticamente significativas $(p>0,05)$. Esto nos demostró, al igual que Peck, que la remode- 
lación ósea no depende de género del paciente sino de su actividad muscular (3).

La presente investigación evaluó el dolor, sin embargo este dato se obtuvo en forma subjetiva al interrogar al paciente durante el examen tomográfico por la presentaba sintomatología dolorosa y a que lado. Es decir, no utilizamos ninguna escala especial de clasificación, sino que reportamos lo que el paciente mencionó durante el examen. Los datos que encontramos en la presente investigación fueron bastante interesantes. Los pacientes que presentaban dolor en el lado derecho se compararon con la longitud del ala externa de la apófisis pterigoides, encontrando relación estadísticamente significativa de mayor longitud en el mismo lado $(\mathrm{p}<0,001)$. Lo mismo sucedió al comparar pacientes con longitud mayor en el lado izquierdo y dolor en el mismo lado $(\mathrm{p}=0,002)$. Estos datos de relación entre dolor y longitud del ala externa de la apófisis pterigoides no han sido reportados en la literatura.

Sato mencionó que la causa de dolor en los pacientes con disfunción temporomandibular, se debe a la presión que se produce sobre el componenete neurovascular de la zona bilaminar, ya que éste se desplaza anteriormente entrando en relación íntima con la superficie articular del cóndilo mandibular (9). La aparición de dolor en el lado que tenemos alargamiento del ala externa de la apófisis pterigoides, se podría explicar debido a que este alargamiento lo hemos asociado con un espasmo crónico del músculo pterigoideo lateral, el cual produciría un desplazamiento discal que reubicaría la zona bilaminar en una posición más anterior. Esta nueva posición de la zona bilaminar podría producir dolor, ya que a boca cerrada el cóndilo mandibular la presionaría contra la cavidad glenoidea del temporal.

Los hallazgos de este estudio, pueden abrir una nueva línea de investigación en nuestra Universidad. Kannan y Sharma reportaron una nueva técnica quirúrgica denominada Disyunción Pterigoidea, en la cual fracturan el ala externa de la apófisis pterigoides, para disminuir o eliminar el dolor articular en pacientes con DTM $(1,2)$. La presente investigación ha encontrado que las alas externas de las apófisis pterigoides en los pacientes con DCM se encuentran alargadas. Si unimos ambos investigaciones se podría desarrollar un proyecto en el cual los pacientes con
DTM, que tengan el ala externa de la apófisis pterigoides alargada, dolor, y hallan sido sometidos a varias terapéuticas para problemas de DTM sin encontrar solución; sean sometidos a esta nueva técnica quirúrgica para solucionar su afectación.

\section{CONCLUSIONES}

Se concluye que la longitud del ala externa de la apófisis pterigoidesse encuentra alargada en los pacientes con Disfunción Temporomandibular en comparación a los pacientes sin disfunción temporomandibular. Por tal motivo, la Disfunción Temporomandibular afectaría las dimensiones de la apófisis pterigoides.

\section{CORRESPONDENCIA}

Jorge A. Beltrán Silva

Av. Honorio Delgado 430 San Martín de Porres, Lima-Perú

Teléfono: 51-994155860

Correoelectrónico: jorge.beltran@upch.pe

\section{REFERENCIAS BIBLIOGRÁFICAS}

1. Kannan S. PterygoidDysjunction: New Minimally invasive technique for the treatment of painful temporomandibular joint dysfunction. J Craniofac Surg. 2010;21: 1264-9.

2. Sharma R. Pterygoid disjunction for internal derangement of temporomandibular joint. J Maxillofac Oral Surg. 2011;10(2):142-7.

3. Peck Ch, Hannam A. Human jaw and muscle modelling. Arch Oral Biol. 2007;52: 300-4.

4. Grunheid T, Langenbach G, Korfage J, Zentner A, Van Eijden T. The adaptive response of jaw muscle to varying functional demands. EuropeanJournal of Orthodontics. 2009; 31: 596-612.

5. Ludlow JB, Ivanovic M. Comparative dosimetry of dental CBCT devices and 64-slice CT for oral and maxillofacial radiology. Oral Surg Oral Med Oral Pathol Oral RadiolEndod. 2008;106(1):106-14.

6. Ueki K, Hashiba Y, Marukawa K, Nakagawa K, Okabe $\mathrm{K}$, Yamamoto E. Determining the anatomy of the descending palatine artery and pterygoid plates with computed tomography in Class III patients. J Craniomaxillofac Surg. 2009;37:469-73.

7. Gidarakou I, Tallents R, Kyrkanides S, Stein S, Moss M. Comparison of skeletal and dental morphology in asymptomatic volunteers and symptomatic patients with bilateral degenerative Joint disease. Angle Orthod. 
$2003 ; 73: 71-8$.

8. Trpkova B, Major P, Nebbe B, Prasad N. Cranio facial asymmetry and temporomandibular Joint internal derangement in female adolescents: a posteroanteriorcephalometric study. Angle Orthod. 2000;70:81-8.
9. Sato S, Kawamura H, Motegi K, et. al. Morphology of the mandibular fossa and articular eminence in temporomandibular joints with anterior disc displacement. Int J Oral Maxillofac Surg. 1996;25:236-8.

Recibido : 06-03-2013

Aceptado: 21-06-2013 